\title{
IMPACTO A CORTO PLAZO DE INTERVENCIÓN DE ENFER- MERÍA EN LA ESTRATEGIA EDUCATIVA DE NUTRICIÓN PARA ESCUELAS SALUDABLES EN ESTUDIANTES DE PRI- MARIA 2012
}

\section{SHORT-TERM IMPACT OF INTERVENTION OF NURSING ON THE EDUCATIONAL STRATEGY OF NUTRITION FOR HEALTHY SCHOOLS IN STUDENTS OF PRIMARY IN 2012}

\author{
María Alejandra Barbosa*, Corina Murcia*, Adriana Maritza Segura*, \\ Jennifer Magaly Silva*, Aida Nery Figueroa**
}

\begin{abstract}
Resumen
Objetivo. Determinar el impacto a corto plazo de una intervención de Enfermería en estudiantes de primaria a través de la medición de conocimientos y comportamientos tendientes a fortalecer hábitos saludables y reducir el riesgo de obesidad desde la infancia.

Método. Estudio pre-experimental, diseño preprueba-posprueba con un solo grupo, 109 estudiantes y 5 docentes, mediante cuestionario autodiligenciado, utilizando SPSS versión 19 para procesamiento de información.
\end{abstract}

Resultados. Se identificó sobrepeso $10 \%$ y obesidad 2\%; impacto positivo en el conocimiento sobre hábitos alimenticios $(15,6 \%)$ y actividad física $(15,7 \%)$; cambios de comportamiento relacionados con actividades del tiempo libre, sin embargo, no fueron estadísticamente significativos. Las conductas alimenticias de docentes se consideran saludables.

Conclusiones. Se recomienda continuidad y permanencia del proceso en la escuela, familia y comunidad, donde el Profesional de Enfermería desempeñe un papel fundamental para la estrategia Escuela Saludable.

Palabras clave: Conocimiento, comportamiento, hábitos alimenticios, actividad física, enfermería.

\section{Abstract}

Objective: To determine the short-term impact of nursing intervention on elementary students through measuring knowledge and behaviors designed to build healthy habits and reduce the risk of obesity from childhood.

* Estudiantes de Enfermería. Universidad Surcolombiana. Neiva, Huila, Colombia.

** Magíster en Enfermería Atención al adulto y anciano.Profesora Facultad de Salud. Email: ainefic@usco.edu.co 
Methods: A pre-experimental study, pretest posttest design with a single group, 109 students and 5 teachers, through a questionnaire filled out by themselves, using SPSS version 19 for information processing.

Results: $10 \%$ overweight and $2 \%$ obesity were identified, as well as a positive impact on knowledge about eating habits (15.6\%) and physical activity (15.7\%), behavioral changes related to leisure activities, however, these were statistically insignificant. The eating behaviors of teachers are considered healthy.

Conclusions: The continuity and permanence of this process in the school, the family and the community is recommended, where the nursing professional plays a key role for the strategy Healthy School.

Key words: Knowledge, Behavior, Eating Habits, Physical Activity, Nursing.

\section{Introducción}

La Organización Mundial de la Salud (OMS) afirma que en el mundo hay más de 346 millones de personas con diabetes; con sobrepeso mil millones de adultos y 42 millones de niños menores de cinco años y la cifra de obesidad supera los 300 millones. La Obesidad es «una enfermedad crónica de Salud Pública, la cual es causa directa de enfermedades cardíacas, circulatorias, colesterol alto, estrés, depresión, hipertensión, cáncer, diabetes, artritis, entre otras, todas ellas aumentando considerablemente la tasa de mortalidad de los colombianos». Esta, junto a la Diabetes Mellitus tipo 2 (DM Tipo 2), la Hipertensión Arterial (HTA) y la Insuficiencia Renal, constituyen una problemática mundial y son patologías que generan altos costos para los países, especialmente al sistema de salud.

En Colombia, según la ENSIN 2010, el 5.2\% de los niños y niñas menores de cinco años son obesos; en cuanto a la población de 5 a 17 años, el 17.5\% presentaron exceso de peso, de los cuales $13.4 \%$ tenían sobrepeso y $4.1 \%$ obesidad. En adultos, las cifras aumentaron de un $46 \%$ en el 2005 a un $51.1 \%$ en el 2010, igualmente se evidenció en el departamento del Huila, en forma más alarmante, de $46 \%$ a $55 \%$ para los mismos años, indicando agudización de la problemática. Asimismo, las enfermedades cardiovasculares continúan ocupando los primeros lugares en morbi-mortalidad a nivel regional, nacional e internacional.

Desde sus inicios, el ser humano ha asumido estilos de vida, que aún sin saberlo, actúan en beneficio o en contra de su salud, convirtiéndose en factores protectores y factores de riesgo respectivamente; dichos estilos de vida comprenden costumbres y hábitos asociados a la alimentación, higiene personal, actividad física, consu- mo de sustancias tóxicas, relaciones interpersonales, comportamiento sexual, uso del tiempo libre, entre otros.

En ese sentido, la promoción de la salud facilita el desarrollo humano y el mejoramiento de la calidad de vida de las personas. Es importante tomar conciencia desde temprana edad, como estrategia para el mantenimiento de la salud, incluyendo las medidas encaminadas a controlar los factores de riesgo y, por ende, el desarrollo de enfermedades crónicas no transmisibles, siendo la infancia una etapa de aprendizaje donde se adquieren no solo conocimientos sino actitudes, hábitos y prácticas que van formando la esencia y personalidad del ser humano.

Es por eso que la escuela representa el escenario propicio para la implementación de la estrategia, teniendo en cuenta que en 1995 la OPS hizo el lanzamiento oficial en Colombia de la Iniciativa Regional de Escuelas Promotoras de la Salud, IREPS, conocida en nuestro país como Escuela Saludable, dirigida a facilitar la promoción y la educación para la salud con enfoque integral en el ámbito escolar.

Lo anterior conllevó a la elaboración, en el 2005 y 2010, de la "Cartilla de Nutrición para Escuelas Saludables», avalada por la Secretaría de Salud y Educación del Huila y Ministerio de Salud y Protección Social, dirigida a docentes, niños, niñas y comunidad académica de los centros educativos del departamento, que invita a involucrarse en el proceso de formación de estilos de vida saludables, en la vivencia de los valores y la generación de actitudes que favorezcan la calidad del ambiente, el crecimiento y desarrollo armónico a nivel personal y social de la comunidad.

Sin embargo, a pesar de su existencia y teniendo en cuenta el aumento de la problemática de la obesidad y 
sobrepeso, se ve la necesidad de la intervención del profesional de Enfermería en el fortalecimiento y continuidad del desarrollo de la estrategia educativa.

Por tal motivo, el presente estudio fue elaborado con el fin de determinar el impacto a corto plazo de la intervención de Enfermería en la estrategia educativa de Nutrición para Escuelas Saludables en estudiantes de básica primaria El Pital, Huila, a través de la medición de conocimientos y comportamientos tendientes a fortalecer hábitos saludables y reducir el riesgo de obesidad desde la infancia.

\section{Metodología}

El presente estudio es de enfoque cuantitativo, de tipo preexperimental, diseño de preprueba - posprueba con un solo grupo, que implica la medición de conocimientos y comportamientos previos y posteriores a una intervención, de corte longitudinal, por lo que se recolecta la información en dos etapas: antes y después de la intervención.

La población escogida fue de 263 estudiantes y 11 docentes de los grados $3^{\circ}, 4^{\circ}$ y $5^{\circ}$ de básica primaria de la Institución Educativa Promoción Social del municipio de El Pital - Huila - Colombia, se obtuvo una muestra de 114 personas, para la cual se utilizó el muestreo probabilístico, de tipo aleatorio simple. Teniendo en cuenta como criterios la participación en las dos etapas de la investigación: previa y posterior a la intervención de enfermería, la presentación del consentimiento informado firmado por sus representantes legales y la ausencia de alguna alteración mental y/o cognitiva.

Para la recolección de la información se utilizó como instrumento un cuestionario autodiligenciado de 14 preguntas, elaborado por las autoras, que busca medir conocimientos y algunos comportamientos de la población en cuanto a nutrición y actividad física, diligenciado antes y después de la intervención por los estudiantes y solo en la fase previa por los docentes, con el fin de medir el impacto de la misma.

Antes de ser aplicado, el instrumento fue sometido a prueba de validez a cargo de tres expertos en el tema, quienes se encargaron de evaluar la pertinencia, redacción, claridad y coherencia, y una prueba de confiabilidad que se realizó mediante una prueba piloto con doce estu- diantes de la Escuela Primaria Ángel María Paredes - Sede Calixto Leiva de la ciudad de Neiva, los cuales se seleccionaron por presentar características similares a la población de estudio.

La tabulación de la información se realiza mediante una base de datos previamente elaborada en el programa Excel versión 2007 y el uso del programa estadístico SPSS versión 19, mediante el cual se realizó un análisis univariado y multivariado. Los resultados se presentan a través de gráficas y tablas, utilizando $x^{2}$ con estadístico Mc Nemar con una confiabilidad del $95 \%$, el cual se emplea para medir el cambio en la población a consecuencia de la intervención.

Se respetan los principios éticos de la Profesión de Enfermería y la Investigación, estipulados en la Ley 911 de 2004 y Resolución 8430 de 1993 respectivamente; la última la clasifica como una investigación sin riesgo. Además, fue aprobado por el Comité de Ética de la Facultad de Salud de la Universidad Surcolombiana.

Adicionalmente, se contó con el aval de la autora de la Cartilla de Nutrición para Escuelas Saludables y, a su vez, de la Secretaría de Salud Departamental del Huila.

\section{Resultados}

El $65 \%$ de la población objeto de estudio se encuentra en edades entre 9 a 11 años, seguido del $24 \%$ entre 6 a 8 años y $11 \%$ mayores de 11 años. El $47 \%$ de la población se encuentra en el grado tercero, el $27 \%$ en grado cuarto y el $26 \%$ en grado quinto. El $56 \%$ de la población corresponde al género masculino y el $44 \%$ al género femenino.

El Índice de Masa Corporal fue adecuado para la edad en un $72 \%$. Sin embargo, se observó sobrepeso en un $10 \%$ y presencia de obesidad en el $2 \%$.

En cuanto a conocimiento sobre obesidad (tabla 1), se logró observar un aumento estadísticamente significativo $\left(\mathrm{X}^{2}=16.5\right.$ y $\left.\mathrm{P}<0.01\right)$ luego de la intervención realizada a los estudiantes de básica primaria.

Igualmente para el concepto de ejercicios aeróbicos y anaeróbicos con un aumento del 15,7\% (gráfica 1).

En el comportamiento, con relación al consumo de alimentos saludables (jugo natural y frutas) y no saludables 
Tabla 1. Conocimiento previo y posterior del concepto de obesidad

\begin{tabular}{|c|c|c|c|c|c|c|}
\hline \multirow{2}{*}{\multicolumn{2}{|c|}{ Concepto }} & \multicolumn{3}{|c|}{ Posterior } & \multirow{2}{*}{$x^{2}$} & \multirow{2}{*}{$\mathbf{P}$} \\
\hline & & Si & No & Total & & \\
\hline \multirow[t]{3}{*}{ Previo } & $\mathrm{Si}$ & 60 & 8 & 68 & \multirow[t]{3}{*}{16,5} & \multirow[t]{3}{*}{$<0,01^{\prime}$} \\
\hline & No & 36 & 5 & 41 & & \\
\hline & Total & 96 & 13 & 109 & & \\
\hline
\end{tabular}

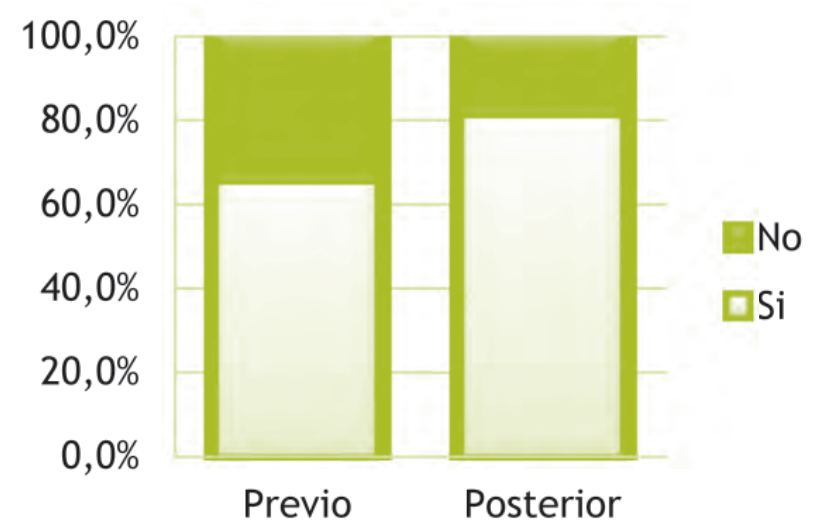

Gráfica 1. Conocimiento previo y posterior sobre ejercicios aeróbicos y anaeróbicos (dulces, papas fritas, empanadas y bebidas gaseosas) a la hora de recreo (tabla 2), se observa que los cambios no fueron estadísticamente significativos $\left(X^{2}<3.86\right.$ y $\left.P>0.05\right)$.

Entre las actividades que realizan con mayor frecuencia los escolares a la hora de recreo, de acuerdo a los resultados previos y posteriores a la intervención, se encuentran microfútbol, la lleva y el escondite.

En cuanto al comportamiento en el hogar, previo a la intervención, teniendo en cuenta el primer vagón de cereales, raíces, tubérculos y plátanos, el $87 \%$ afirmó consumir este tipo de alimentos, entre ellos el principal fue el pan (ver gráficas 2 y 3 ).

Tabla 2. Comportamiento previo y posterior relacionado con hábitos alimenticios a la hora de recreo

\begin{tabular}{|c|c|c|c|c|c|c|}
\hline \multirow{2}{*}{\multicolumn{2}{|c|}{$\begin{array}{l}\text { Consumo de alimentos } \\
\text { a la hora del recreo }\end{array}$}} & \multicolumn{3}{|c|}{ Posterior } & \multirow{2}{*}{$\mathrm{X}^{2}$} & \multirow{2}{*}{$\mathbf{P}$} \\
\hline & & Si & No & Total & & \\
\hline \multicolumn{7}{|l|}{ Jugo } \\
\hline \multirow[t]{3}{*}{ Previo } & $\mathrm{Si}$ & 58 & 23 & 81 & 1,7 & 0,18 \\
\hline & No & 14 & 14 & 28 & & \\
\hline & Total & 72 & 37 & 109 & & \\
\hline \multicolumn{7}{|l|}{ Dulces } \\
\hline \multirow[t]{3}{*}{ Previo } & $\mathrm{Si}$ & 41 & 24 & 65 & 0,25 & 0,65 \\
\hline & No & 20 & 24 & 44 & & \\
\hline & Total & 61 & 48 & 109 & & \\
\hline \multicolumn{7}{|c|}{ Empanada } \\
\hline \multirow[t]{3}{*}{ Previo } & $\mathrm{Si}$ & 25 & 22 & 47 & 0,22 & 0,63 \\
\hline & No & 18 & 44 & 62 & & \\
\hline & Total & 43 & 66 & 109 & & \\
\hline
\end{tabular}




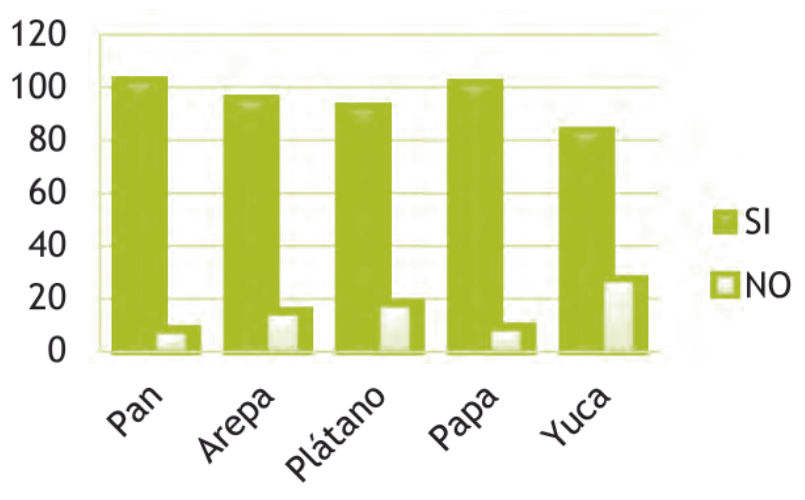

Gráfica 2. Alimentos que consumen en el hogar, del Vagón 1 del Tren de la Alimentación

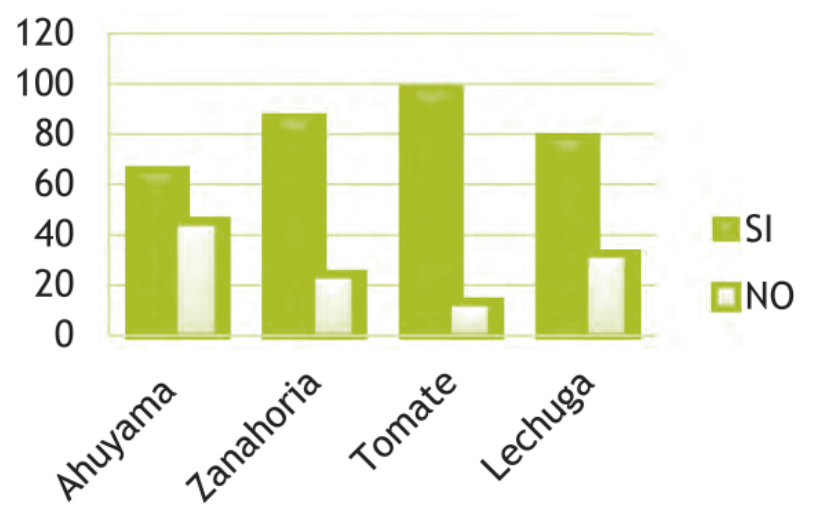

Gráfica 3. Alimentos que consumen en el hogar, Vagón 2 del Tren de la Alimentación

En cuanto al segundo vagón de hortalizas, verduras y leguminosas secas, el 75\% respondió incluir estos alimentos dentro de sus comidas, el de mayor consumo fue el tomate.

El $90 \%$ consumen en el hogar frutas como el banano, la mandarina, el mango, la granadilla y de estos el de mayor consumo fue el banano. El 87\% afirma incluir carnes, huevos y leguminosas secas dentro de su alimentación, 13\% aseguraron no consumirlos y el alimento de mayor consumo es el huevo (ver gráfica 4).

El consumo de lácteos representa el 82\%, siendo el de mayor frecuencia la leche. El $58 \%$ de los escolares afirmaron incluir las grasas en su alimentación. Se destaca el consumo de la mantequilla y el aceite. El $72 \%$ de los niños asegura consumir azúcares y dulces en el hogar (ver gráficas 5 y 6 ).

En cuanto a hábitos alimenticios de los docentes, durante el período de descanso de la jornada laboral, los alimentos que más consumen son el café, jugo natural y frutas; empanada o pastel y sándwich también son con-

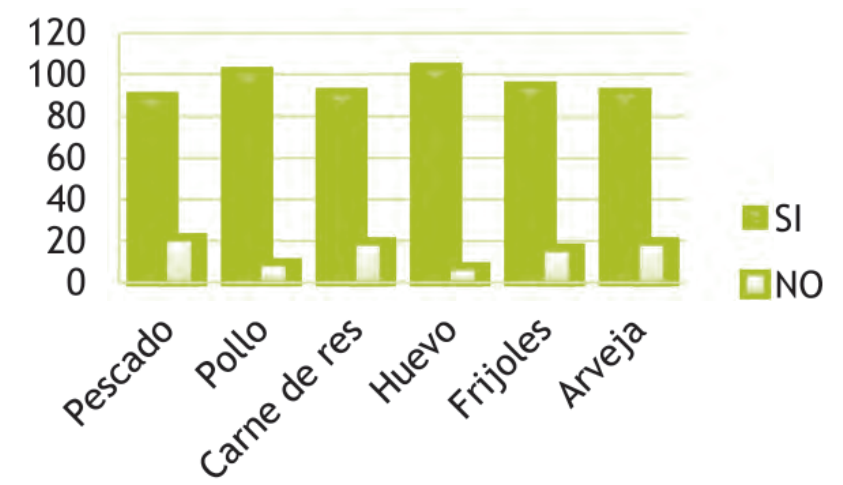

Gráfica 4. Alimentos que consumen en el hogar del Vagón 4 del Tren de la Alimentación

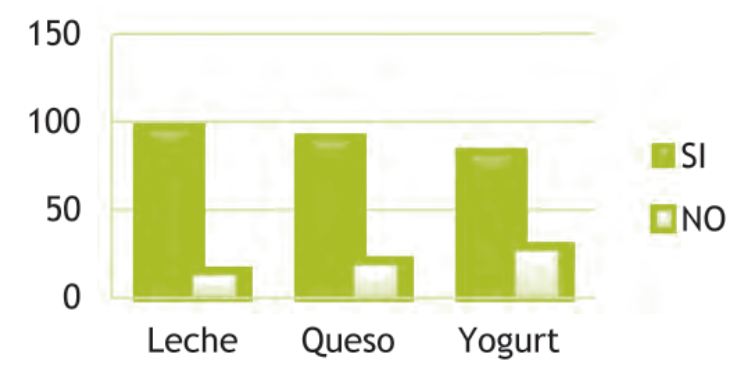

Gráfica 5. Alimentos que consumen en el hogar del Vagón 5 del Tren de la Alimentación

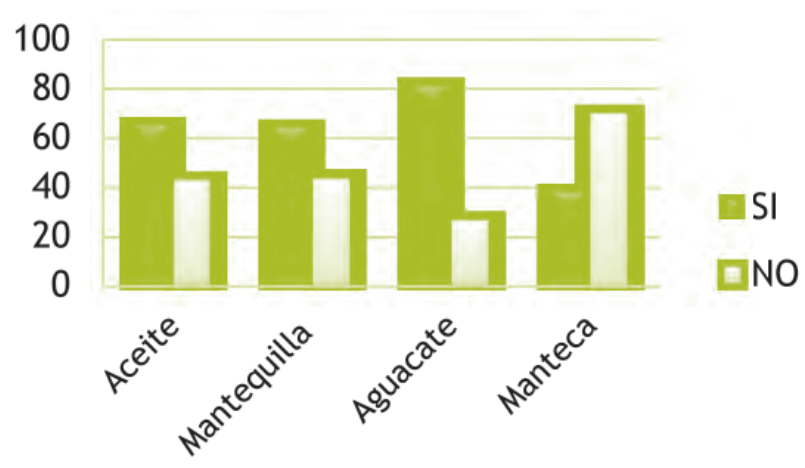

Gráfica 6. Alimentos que consumen en el hogar, Vagón 6 del Tren de la Alimentación

sumidos pero en menor frecuencia. Se observó no consumo de bebidas gaseosas. Con respecto a las actividades que realizan los docentes de básica primaria, se puede resaltar la falta de práctica de deportes como fútbol, voleibol y baloncesto.

\section{Discusión}

La escuela, como centro de formación integral, constituye el espacio propicio para la adquisición de 
conocimientos por parte de la población infantil, la cual se encuentra en una etapa del ciclo vital caracterizada por la maduración de las habilidades motoras y mayor desarrollo cognitivo, lo cual para Bueno, Sarría y Pérez (2003) permite establecer patrones de alimentación, hábitos, gustos y desagrados, muchos de los cuales van a persistir durante el resto de sus vidas.

Por tal motivo, el conocimiento es una herramienta fundamental para la toma de decisiones orientada hacia estilos de vida saludables y constituye un factor protector en la prevención de la obesidad. Por ello es importante que los estudiantes alcancen y mantengan niveles de conocimiento que vayan a la par con su comportamiento. Según lo planteado por Kain, Olivares, Castillo y Vio (2001), para lograr los cambios conductuales necesarios para prevenir la obesidad, se requiere en primer lugar adquirir los conocimientos básicos en estos aspectos.

Esto concuerda con los resultados de la presente investigación, teniendo en cuenta el cambio positivo obtenido en el conocimiento, es posible lograr cambios en el comportamiento, aunque este no fue estadísticamente significativo, como en el caso de los docentes, que tan solo dos de ellos montan bicicleta una o dos veces a la semana, siendo necesario un seguimiento e incluir a la familia y la comunidad para el fomento de estilos de vida saludables basados en buenos hábitos alimenticios y práctica frecuente de actividad física para evitar el sedentarismo y prevenir con ello la obesidad.

De acuerdo con María Castillo (2007), la escuela, además de transmitir conocimientos, colabora en el desarrollo psíquico y personal de los niños y niñas, siendo la salud el bien más preciado y un derecho fundamental. Por lo tanto, especialmente en básica primaria, el docente constituye el referente principal para el estudiante, siendo un modelo a seguir e influye en su conducta.

El impacto positivo de esta estrategia en el conocimiento y en menor medida en el comportamiento indica que la intervención de Enfermería con la «Cartilla de Nutrición para Escuelas Saludables» debe tener un seguimiento periódico, además del trabajo intersectorial que involucre al sector salud y educativo, para lograr efectos positivos a mediano y largo plazo cuando los niños conozcan cuáles son los alimentos que deben consumir, en qué proporción y adquieran conductas saludables de acuerdo a su edad como lo afirma la Organización Mundial de la Salud.
Así como la institución educativa cumple un papel importante en la formación integral del ser humano, la familia constituye parte esencial en el desarrollo de actitudes y conductas en los hijos, especialmente en la etapa escolar, donde asumen comportamientos de la vida diaria y son influenciados por el entorno, razón por la cual es importante que cada miembro de la familia fomente estilos de vida saludables. De este modo, Fernández y López (2010), resaltan la implicación de la familia especialmente en programas de modificación de conducta en los que los padres actúan como agentes de cambio, modificando sus propios estilos de vida, resultando más efectivo que los dirigidos únicamente a los cambios de comportamiento en los niños/as.

Lo anterior confirma la importancia de aplicar esta intervención en la familia y toda la comunidad educativa, como lo aseguran Prada y Herrán (2009), el núcleo familiar y el entorno social son de mayor influencia en la formación de hábitos alimentarios y tienen una participación trascendental cuando se busca promocionar hábitos en los preescolares y escolares.

De igual manera, en la teoría del Autocuidado de Dorotea Orem, los niños son agentes de cuidado dependiente, por lo que ellos no son totalmente responsables de ciertos patrones de conducta; de allí que su formación debe incluir a la familia, la escuela y la comunidad en general, sin dejar de lado la responsabilidad del cuidador en cuanto a la enseñanza del autocuidado en su hijo. Asimismo, el Profesional de Enfermería cumple un papel fundamental en este proceso, ya que su esencia es el cuidado integral del individuo en todas sus dimensiones.

Se resalta la importancia de la elaboración de una estrategia educativa, donde además de hábitos alimenticios se incluya actividad física, con el fomento de actividades que impliquen un gasto de energía, así como lo indica Kain, Olivares, et al. (2001), en su estudio, donde demuestran la efectividad de una intervención que incluye educación en alimentación, nutrición y aumento de la actividad física.

\section{Conclusiones}

En la fase previa, a nivel general, se observó desconocimiento de los contenidos de la Cartilla de Nutrición para Escuelas Saludables, relacionados con hábitos alimenticios y 
actividad física. Sumado a esto, los comportamientos incluían un desequilibrio en el consumo de los alimentos de cada vagón así como alimentos no saludables en la hora de recreo.

En la fase posterior a la aplicación de la estrategia educativa, el nivel de conocimiento mejoró al obtener que un $81.7 \%$ de los estudiantes lograran reforzar su conocimiento. Sin embargo, no se logró observar cambios significativos en el comportamiento. Uno de los factores que podría limitar este cambio son los alimentos no saludables que ofrecen las tiendas escolares, tales como refrescos, gaseosas, dulces, papas fritas, empanadas, entre otros. Debido a ello, es importante que los procesos de formación educativa, como la aplicación de dicha estrategia, sean continuos y con mediciones frecuentes, donde se promueva la participación activa tanto de los docentes como del profesional de Enfermería en este proceso.

Al comparar las fases previa y posterior a la intervención, los resultados demuestran que la estrategia educativa «Cartilla de Nutrición para Escuelas Saludables» tuvo impacto positivo solo a nivel de conocimiento relacionado con hábitos alimenticios y actividad física.

Las conductas del niño están influenciadas desde su entorno familiar, donde los hábitos alimenticios se ven afectados por factores socioeconómicos principalmente y algunas características propias del municipio como la agricultura, además de la cultura y la idiosincrasia.

Se identificó en los docentes el consumo de alimentos saludables y predominio de actividades sedentarias. Es importante tener en cuenta que los docentes son los modelos educativos de los escolares y, por tanto, deben brindarles buenas bases de aprendizaje, especialmente en hábitos alimenticios y actividad física, por lo que su conducta alimenticia y, en general, sus estilos de vida son de gran influencia en la formación de los niños.

\section{Agradecimientos}

A la Secretaría de Salud Departamental del Huila, por dar el aval para el uso del material de su autoría, «Cartilla de Nutrición para Escuelas Saludables».

Al Programa de Enfermería de la Universidad Surcolombiana, por el apoyo y acompañamiento. Igualmente, a la Institución Educativa Promoción Social- Sede Primaria del Pital - Huila y a toda la comunidad educativa.

\section{Referencias bibliográficas}

Organización Mundial de la Salud.

Instituto colombiano de Bienestar Familiar. Encuesta Nacional de la Situación Nutricional. Bogotá, p. 381, 2005.

Lalonde, Marc. Una nueva perspectiva en la salud de los canadienses. Canadá, 1974.

República de Colombia- Ministerio de Salud. Resolución 8430 (4, octubre, 1993). Por la cual se establecen las normas científicas, técnicas y administrativas para la investigación en salud. Bogotá, D.C.; Diario Oficial, 1993.

Trujillo, Claudia. Cartilla de Nutrición para Escuelas Saludables. Neiva, Huila: Secretaría de Salud Departamental. Cuarta Edición, 2010.

Bueno, M, Sarría, A y Pérez, JM. Nutrición en pediatría. 2a edición. Editorial Ergon, 2003.

Olivares, S, et al. Validación y aplicación de instrumentos para evaluar intervenciones educativas en obesidad en escolares. En: Revista Chilena de Pediatría, 72(4) (2001):308-18.

Castillo, María José. Prevención de la obesidad infantil desde la escuela. Revista digital «Práctica Docente». Núm. 7, 2007.

Fernández, Juan Pablo y López Ricardo. El sobrepeso y la obesidad en edad escolar. Estudio y variaciones del IMC en una población de educación secundaria durante un curso escolar. Revista Digital, Buenos Aires, Núm. 144, Mayo de 2010.

Prada, Gloria y Herrán, Oscar. Impacto de estrategias para aumentar el consumo de frutas y verduras en Colombia. Observatorio Epidemiológico de Enfermedades Cardiovasculares. Universidad Industrial de Santander, Bucaramanga, Colombia [online]. En: Revista Chilena de Nutrición, Vol. 36, Núm. 4, 2009.

Raile, Martha \& Marriner, Ann. Modelos y Teorías de enfermería. Barcelona, España: Editorial Elsevier. Quinta Edición, 2002. 